\title{
MAGNETIC FIELD INDUCED LOCALIZATION IN SEMIINSULATING GaAs
}

\author{
J. Lusakowski, R. Merten and M. Grynberg \\ Institute of Experimental Physics, Warsaw University \\ Hoża 69, 00-681 Warszawa, Poland
}

\begin{abstract}
Conductivity experiments were carried out on samples of semiinsulating GaAs at liquid helium temperature in magnetic field up to $6 \mathrm{~T}$. During the measurements the samples were persistently illuminated with infrared light which allowed to populate with electrons a part of shallow donor and conduction band states. Current-voltage characteristics showed an abrupt jump of the current at a threshold electric field which is interpreted as a result of impact ionization of electrons bound on shallow donors and in the tail of the bottom of the conduction band. The jump of the current decreases as the magnetic field increases and disappears for a sufficiently high magnetic field $B_{0}$. The value of $B_{0}$ grows with growing light intensity. These results are explained by magnetic-field induced localization of electrons on long-range fluctuations of the electrostatic potential. The localization transition was confirmed by the current dependence on temperature measured at different magnetic fields. A peak on these curves was observed. Its position coincides with the temperature above which impact ionization is not observed. A possible mechanism explaining appearance of the peak is presented.
\end{abstract}

PACS numbers: $71.55 . \mathrm{Jv}, 72.15 . \mathrm{Gd}, 72.20 \mathrm{Ht}$

\section{Introduction}

Conductivity properties of compensated semiconductors have been a subject of experimental and theoretical investigation for many years. Compensation leads to formation of charged centers randomly distributed in the crystal lattice and thus it is a cause of disorder. Some of fundamental questions in the physics of compensated semiconductors concern the problem of an influence of disorder on the structure of electronic states and localization in such materials [1].

A fluctuating electrostatic potential which results form the spatial disorder can be described by a model proposed by Efros and Shklovskii [2]. Let a sample be divided into spheres with a radius $R$. On average, each sphere will contain $N_{t} R^{3}$ charged centers. However, the exact number of charged centers in each sphere fluctuates with a standard deviation of $\left(N_{t} R^{3}\right)^{1 / 2}$. The long-range potential $\Delta V$ generated by this excess charge within each sphere (Fig. 1a) is thus of the order of $e\left(N_{t} R\right)^{1 / 2}$ and grows with growing $R$. On the other hand, the concentration 


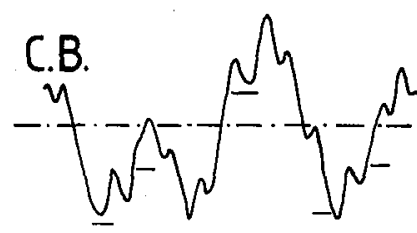<smiles>[CH-]</smiles>
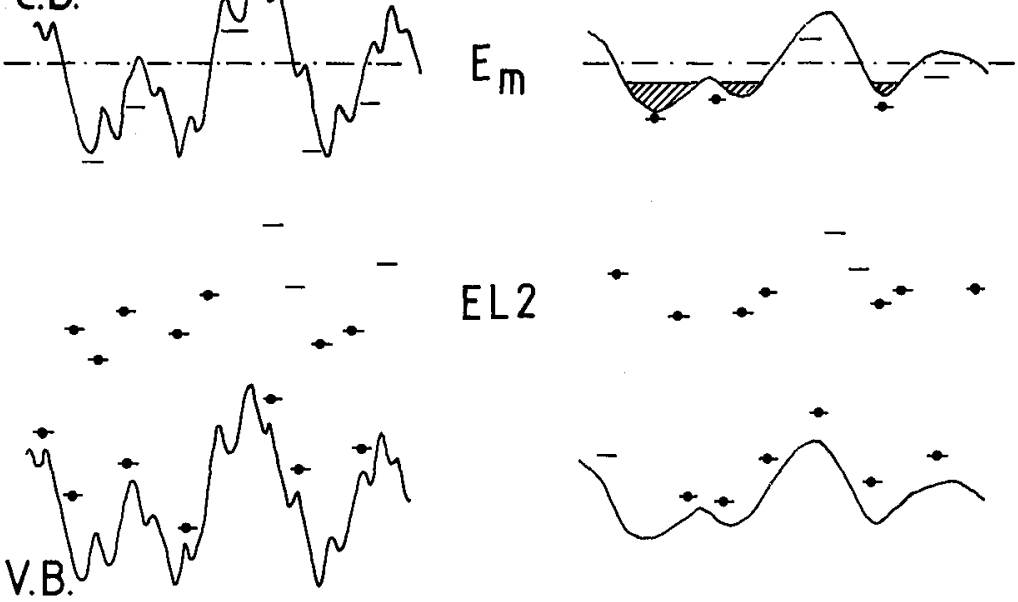

a

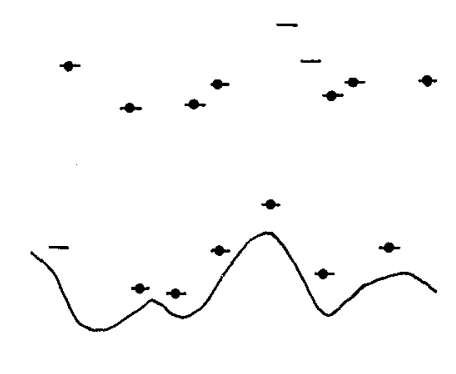

b

Fig. 1. Perforation of the conduction (c.b.) and the valence (v.b.) band caused by potential fluctuations in EL2 rich SI GaAs before (a) and during (b) illumination with infrared light. $E_{\mathrm{m}}$ is the mobility edge.

of the excess charge is equal to $\left(N_{t} R^{-3}\right)^{1 / 2}$ and decreases with increasing $R$. The maximum value of $\Delta V$ is determined by the screening radius $R_{\mathrm{s}}$, i.e. all fluctuations with radii $R>R_{\mathrm{s}}$ are screened out and do not contribute to the fluctuating potential. Usually, screening is thought to be a result of spatial redistribution of free carriers. However, in high-resistivity materials screening can also be accomplished by redistribution of electrons bound on deep or shallow centers [3]. Let $N_{\mathrm{s}}$ be the concentration of all screening charges. The screening radius $R_{\mathrm{s}}$ is determined by the condition $N_{\mathrm{s}}=\left(N_{t} R_{\mathrm{s}}^{-3}\right)^{1 / 2}$, i.e. $R_{\mathrm{s}}=\left(N_{t} N_{\mathrm{s}}^{-2}\right)^{1 / 3}$. This result should be applied with caution for it was derived on the assumption of statistically random distribution of charged centers. Since correlations of the locations of dopants [4] and native defects $[5,6]$ may arise during the crystal growth process or during illumination of the sample the above formula may lead to misleading results even if the screening concentration $N_{\mathrm{s}}$ were known.

The measurements described in this paper were carried out on samples of semiinsulating (SI) undoped GaAs which electrical and optical properties are largely controlled by EL2 [7], a native midgap level which overcompensates other donor and acceptor centers. If a sample of this material is cooled to liquid helium temperature then one can safely say that the concentration of free electrons is zero and all the shallow donors and shallow acceptors are ionized. The Fermi level is pinned by the EL2 centers, some of which are ionized. In a thermal equilibrium charged and neutral EL2 are distributed so as to minimize the Coulomb energy of the system. A distribution of charged centers defines the screening radius. Thus, in such conditions, screening is accomplished by localized charges only. 
To enable conductivity measurements at liquid helium temperature the samples were persistently illuminated during the experiment with low-intensity light of the photon energy of $1.45 \mathrm{eV}$. This energy corresponds to slightly less than the band gap energy and is clear of the intra-EL2 bleaching transition [7]. This illumination, which excites electrons from EL $2^{\circ}$ states and possibly other band gap levels, creates a steady-state population of shallow donor and conduction band states. The process of excitation is stochastic which means that new charged centers appear at randomly scattered places in a sample and the fluctuating potential pattern may change in time. In spite of this, it seems correct to assume that the statistical distribution of the fluctuating fields is time independent. It follows that the measured quantities (current, voltage) are time-a veraged over periods of time long compared with a time of excitation of an electron by the pumping light.

A lifetime of an optically pumped electron on a shallow donor and in the conduction band is long enough to enable conductivity measurements. This can be explained by the configurational barrier of the EL2, which makes direct recombination of an electron photoexcited from this state difficult. An additional factor which increases the electron lifetime are the potential fluctuations; after an electron is photoexcited from a deep center high into the conduction band it thermalizes into the deepest available well of the potential relief and thus finds a location which is usually spatially well separated from the parent deep center.

A stationary concentration of neutral shallow donors $\left(n_{\mathrm{d}}\right)$ and free electrons (n) can be found from a set of rate equations. A very simple model of this kind was analyzed. It look into account only thermal generation and recombination between shallow donors and the conduction band and optical excitation and thermal recombination between the EL2 and the conduction band. Since these are the most relevant processes which define the stationary state of the system this model can be regarded as a first approximation of a more complicated description. In advantage it gives possibility of obtaining analytical solutions. It was found that for low illumination intensity both $n_{\mathrm{d}}$ and $n$ are proportional to the light intensity. This result will be used below.

One can expect that under the illumination the amplitude of the fluctuating potential decreases (Fig. 1b). This comes from the fact that photoexcited electrons fill first the deepest wells of the potential relief and partially neutralize them. Second, the illumination creates a stationary concentration of free and weakly bound electrons which can shift to the deepest potential wells more easily than deeply bound electrons.

The fluctuating potential broadens the density of shallow donor states and leads to formation of a tail at the bottom of the conduction band [8]. In the presence of spatial disorder the electron states can be divided into localized (lower energy) and extended (higher energy). The value of energy $E_{\mathrm{m}}$ which separates the two types of states is called the mobility edge [9] and coincides with the percolation threshold energy. 


\section{Impact ionization of shallow donor}

Impact ionization of a shallow donor state is a known phenomenon which has been investigated in different semiconductors for more than thirty years [10]. It is a process of ionization of a neutral donor caused by a collision of a free electron with a bound electron. If the electric field applied to a sample is sufficiently high impact ionization leads to multiplication of free electrons. This manifests by a rapid increase of the current at a threshold electric field $E_{\text {th }}$ on a current voltage characteristic [11]. For samples of $n$-type GaAs the value of $E_{\text {th }}$ is typically equal to a few V/cm. Magnetic field $B$ influences impact ionization by shifting $E_{\text {th }}$ to higher values [12]. This is easily understandable because, first, magnetoresistivity lowers the speed of electron heating, second, an increase of the ionization energy of a shallow donor in the magnetic field [13] requires higher energy of a free electron to impact ionize. Up to the authors' best knowledge, very few papers considered impact ionization at high magnetic field, which is of interest of the present work. On the basis of the literature data [12] one can state that the magnetic field as high as $5 \mathrm{~T}$ does not suppress impact ionization.

Le Hir [14] showed results of an investigation of low $B$ influence on impact ionization for a number of Ge:As samples for the magnetic field perpendicular and parallel to the applied electric field. In Fig. 2 some of his results are shown for two

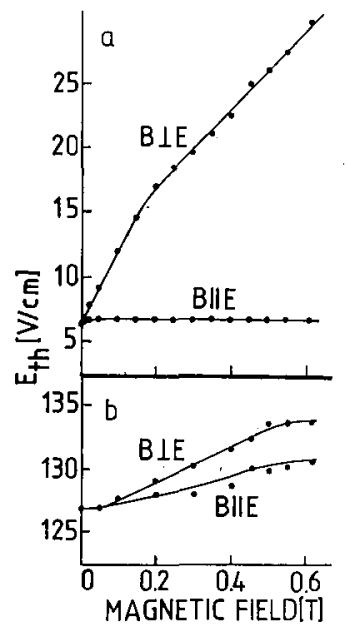

Fig. 2. The threshold electric field $E_{\mathrm{th}}$ as a function of magnetic field for two samples of Ge:As; a) As concentration $N_{\mathrm{As}}=1.4 \times 10^{14} \mathrm{~cm}^{-3}$, compensation $K=0.2$; b) $N_{\mathrm{As}}=3.8 \times 10^{16} \mathrm{~cm}^{-3}, K=0.12$. After [14].

samples with different shallow donor concentrations and a similar compensation. Since the magnetic field is small one may state that the $E_{\text {th }}$ dependence on $B$ is caused only by magnetoresistivity. For the sample with low As concentration the $E_{\text {th }}$ grows with $B$ only for $B$ perpendicular to $E$, which agrees with a simple model predicting zero magnetoresistivity for $B$ parallel to $E$. Ilowever, this is not 
the case for the highly doped sample where two curves almost coincide and show an increase of $E_{\text {th }}$ with $B$. It means that in this case a dominant scattering mechanism is independent of $E$ and $B$ configuration. In other words, the longitudinal and transverse magnetoresistivity are (almost) equal. The independence of magnetoresistivity on $E$ and $B$ configuration is a feature of systems with a fluctuating potential [15]. Thus we conclude that the similarity of the $E_{\text {th }}$ dependence on $B$ for both configurations comes from potential fluctuations present in a highly doped material.

\section{Experimental}

The samples used in the experiments were cut from one wafer of undoped SI-GaAs. The concentration of the EL2 was found from absorption measurements [16] to be equal to $5.3 \times 10^{16} \mathrm{~cm}^{-3}$. Thermally stimulated current measurements showed that other deep levels were also present in the investigated material. The Hall effect measurements gave the value of the electron concentration and mobility to be of the order of $10^{8} \mathrm{~cm}^{-3}$ and $400 \mathrm{~cm}^{2} /(\mathrm{V} \mathrm{s})$ at room temperature, respectively. All the samples were supplied with $\mathrm{Au} / \mathrm{Ge} / \mathrm{Ni}$ contacts wlıch were ohmic down to liquid helium temperatures [17]. A sample was placed in a helium cryostat in a $6 \mathrm{~T}$ superconducting coil and persistently illuminated with infrared light. The sample was shielded against any thermal far infrared radiation; it was cooled by an exchange gas. The temperature of the sample was changed by a resistive heater and it was measured by an Allan-Bradley resistor.

\section{Magnetic field induced localization}

As a representative example of data, in Fig. 3 we present a set of current-voltage characteristics measured as a function of the magnetic field perpendicular to the direction of the external electric field. For low magnetic fields there is a

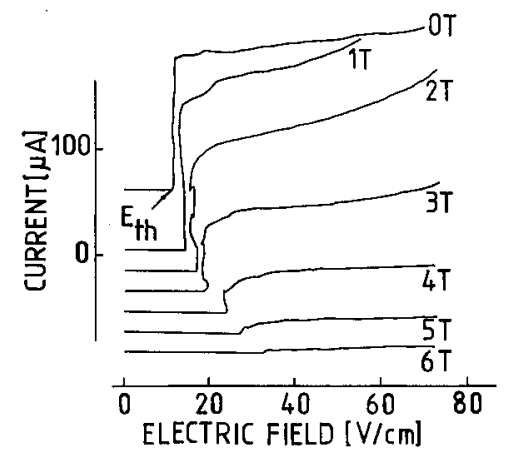

Fig. 3. Current-voltage characteristics as a function of magnetic field.

well-defined threshold value of the electric field $E_{\text {th }}$ for which an abrupt increase of the current is observed. The jump of the current covers three to four orders 
of magnitude for the lowest magnetic fields at the illuminating light intensity used. When the magnetic field is increased the jump of the current becomes less pronounced and finally, for the strongest fields, it disappears. The shape of the $I-V$ curves is exactly the same as observed in $n$-GaAs, where switching between low- and high-conductivity states is caused by impact ionization of the shallow donor states, as described above. Moreover, the observed increase of $E_{\text {th }}$ with increasing $B$ is analogous to that found in $n$-GaAs and the value of $E_{\text {th }}$ is similar to that of $n$-type samples [12]. On that basis we interpret the observed shape of the $I-V$ curves as a result of impact ionization of the shallow donors. An obvious condition for this phenomenon to occur, namely a significant occupation of donor states, was directly confirmed in far infrared magnetooptical experiments [18].

The question however appears, why does the jump of the current decreases when the magnetic field gets stronger? In other words, why does the impact ionization cease to be active above sufficiently high magnetic field (about $6 \mathrm{~T}$ for the presented data)? This is an unexpected result, since for an $n$-type material impact ionization of shallow donors takes place at such magnetic fields. From Fig. 3 one gets that for any electric field greater than $E_{\text {th }}$ the current decreases when the magnetic field grows. It was found that the corresponding increase of resistivity is more rapid than an exponential one. Measurements of $I-V$ characteristics for the magnetic field parallel to the external electric field gave a result identical to that obtained in the perpendicular configuration, which excludes a simple magnetoresistivity model as an explanation of the data. It is also an indication of presence of potential fluctuations in the investigated system.

A strong magnetic field dependence of the resistivity is usually connected with the phenomenon of localization. It seems plausible to consider two types of localization: freeze-out on shallow donors $[8,12]$ and localization on potential fluctuations [19]. However, freeze-out itself does not disturb impact ionization [12], merely shifting the threshold electric field to compensate for an increase of the electron-donor binding energy. To explain the experimental data one has to assume that the electrons cannot gain enough energy from the electric field because they are confined within wells of the fluctuating potential. Thus, we propose that the electron localization on the potential fluctuations is responsible for the observed dependence of current on the magnetic field. It is very probable that freeze-out also takes place, but its influence alone is insufficient to explain the experimental data.

Within this model the disappearance of impact ionization can be described as follows. At zero magnetic field, for electric fields greater than $E_{\text {th }}$ there is a significant concentration of hot electrons with energies above the mobility edge $E_{\mathrm{m}}$. The application of a magnetic field increases the density of states at the bottom of the lowest Landau level [8], causing electrons to occupy states with lower and lower energy. The current then decreases since fewer and fewer electrons can take part in the impact ionization process. Impact ionization vanishes when the concentration of electrons thermally activated above the mobility edge is insufficient to trigger the avalanche. This occurs when the magnetic-field-dependent quasi Fermi level falls below $E_{\mathrm{m}}$ by about $k T$. Afterwards the current is carried by electron hopping on localized states. 


\section{Light intensity and temperature dependencies}

In a series of experiments we investigated the influence of the illuminating light intensity $P$ on the localization process. The value of the magnetic field $B_{0}$ required for the suppression of impact ionization increases with $P$. To explain this behavior we notice that a stronger illumination creates a higher concentration of electrons on shallow donors and in the conduction band. Then, after impact ionization, the number of electrons with energies greater than $E_{\mathrm{m}}$ increases with $P$ and a stronger magnetic field is required to localize them. The concentration of optically excited electrons is proportional to $P$, as it was described above. On the other hand, the density of states at the bottom of the conduction band increases linearly with magnetic field [8]. If one assumes that the mobility edge does not shift with the illumination intensity (at least in the investigated range of $P$ ) the magnetic field $B_{0}$ required for localization should vary linearly with $P$. The results shown in Fig. 4 are consistent with this interpretation. Figure 5 shows

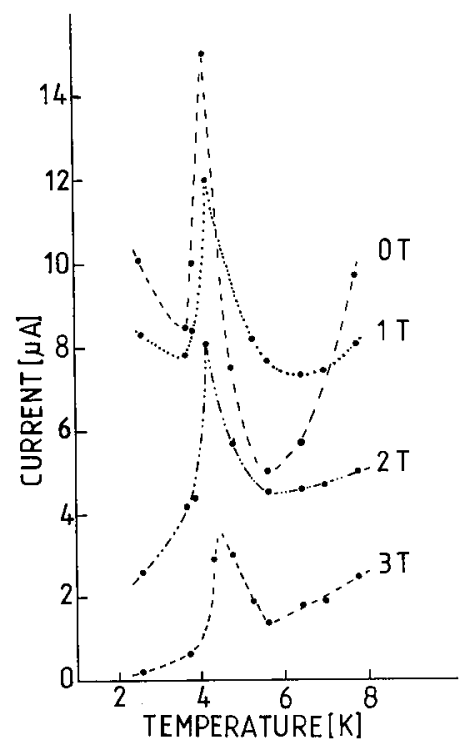

Fig. 4. Temperature dependence of the current for different magnetic fields. The lines are eye guides only.

the dependence of the current on the temperature $T$ for different magnetic fields. These curves were plotted for the same electric field (greater than $E_{\mathrm{th}}$ ) and the same illumination intensity. Let us first focus on the general trend of the curves, neglecting for a while the peak observed about $4.5 \mathrm{~K}$. For the lowest magnetic fields the current depends weakly on temperature. For the magnetic fields stronger than about $2 \mathrm{~T}$ the current dependence on temperature exhibits an activated character which is interpreted as a result of "opening" of an energy gap. A transition from a non-activated to an activated current dependence on temperature is a fingerprint 


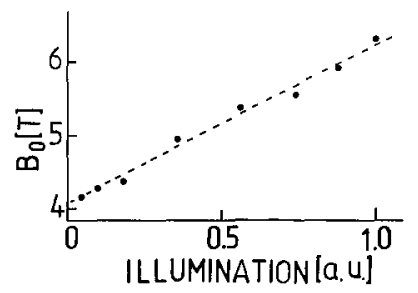

Fig. 5. The magnetic field $B_{0}$ as a function of the illumination intensity.

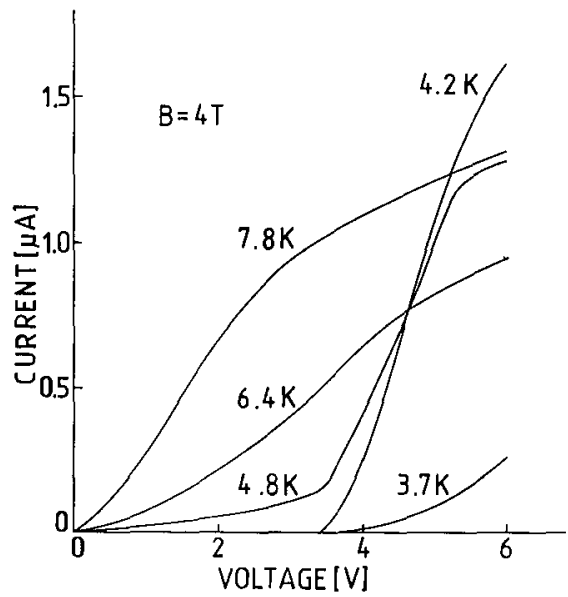

Fig. 6. Current-voltage characteristics for different temperatures at the magnelic field of $4 \mathrm{~T}$.

of metal-insulator transition. The origin of a "metallic" and "insulating" pliases in the investigated system can be understood as follows. For electric fields greater than $E_{\text {th }}$ a coefficient $G$ of the thermal generation of electrons from the shallow donors and the potential wells to the conduction band is negligible when compared to the impact ionization coefficient $A$. Then, for low magnetic fields, the balance between bound and free electrons is not established by thermal processes and the concentration of free electrons weakly depends on temperature. This resembles a metallic behavior. On the other hand, in sufficiently strong magnetic fields the electrons are localized and the number of impact ionized electrons becomes much smaller than that of bound ones, even if $A$ is still much stronger than $G$. Then, it is the thermal generation which essentially influences the free electron concentration and the current shows an activated behavior. The energy gap corresponds to a separation between the quasi Fermi level and the mobility edge and increases with increasing $B$. For the presented data it reaches about $2 \mathrm{meV}$ at $6 \mathrm{~T}$.

Figure 6 shows a set of current-voltage characteristics measured for different temperatures at the magnetic field of $4 \mathrm{~T}$. For $T$ greater than $5 \mathrm{~K}$ the impact ionization is less and less pronounced and disappears for $T$ above about $6 \mathrm{~K}$. This 
is interpreted as a result of thermal ionization of bound electrons. The range of temperature where impact ionization vanishes corresponds to that of the peak of the current in Fig. 5. We propose the following interpretation of this behavior. The peak of the current is observed at temperatures for which an essential part of the electrons is localized but thermal excitations ionize them easily. This is likely to occur when the quasi Fermi level crosses the mobility edge. We suggest that the result of the crossing is similar to that found in IIgSe:Fe when the Fermi level crosses the resonant iron level [20]; correlations between electrons lead to formation of a long-range ordered pattern of localized electrons. This decreases the ionized impurity scattering and leads to an increase of the current.

\section{Conclusions}

Results of conductivity measurements carried out on illuminated samples of SI-GaAs were interpreted on the basis of a model of a compensated semiconductor with long-range fluctuations of the electrostatic potential. The observed vanishing of impact ionization of shallow donors with increasing magnetic field was shown to be caused by electron localization on the potential fluctuations. Impact ionization was proved to be a tool which allowed to discriminate betwcen freeze-out on the shallow donors and localization on the fluctuations.

The results presented above seem to be of a general character. First, one should expect vanishing of impact ionization also for $n$-GaAs but for magnetic fields much stronger than the ones used in these experiments. This should occur when almost all the electrons would be freezed-out on shallow donors. Then, there would be no electrons to trigger the avalanche. A difference between this (possible) phenomenon and the SI-GaAs case lies in the range of the localizing potential; in the former case that would be the potential of a single donor, in the latter this is a long-range fluctuating potential formed by many charged centers. Second, the experiments described above should give similar results when carried out on other high resistivity materials. The only condition is a possibility of creation of a stationary concentration of neutral impurities and free carriers by illumination or other methods.

The described above localization transition is not an equilibrium one. The point is that the electron gas which undergoes localization is driven by the electric field and by the illumination far from the equilibrium. In such conditions the electron temperature is not equal to that of the lattice [8] and the sample response is not a linear function of the applied electric field. Thus, one should not expect that this type of localization would be described by the scaling theory of localization [21].

\section{Aknowledgments}

The authors would like to express their gratitude to Dr. E. Kamińska and Dr. A. Piotrowska, Institute of Electron Technology, Warsaw, for alloying the contacts to the samples. They are thankful to A. Kurpiewski and G. Nowak for carrying out absorption and Hall effect measurements, respectively. Discussions with K. Karpierz and M. L. Sadowski are greatly acknowledged. 


\section{References}

[1] B.I. Shklovskii, A.L. Efros, Electronic Properties of Doped Semiconductors Springer-Verlag, Berlin 1984.

[2] B.I. Shklovskii, A. L. Efros, Zh. Eksp. Teor. Fiz. 60, 867 (1971).

[3] W.G. Karpov, Fiz. Tekh. Poluprovodn. 15, 217 (1981).

[4] L.V. Keldysh, G.P. Proshko, Fiz. Tverd. Tela 5, 3378 (1963).

[5] J. Wagner, W. Wettling, J. Winscheif, W. Rothemund, J. Appl. Phys. 65, (12) 5225 (1989).

[6] M.L. Sadowski, K. Karpierz, M. Grynberg, Phys. Rev. B 43, (9) 7332 (1990).

[7] M. Kamińska, Rev. Phys. Appl. 23, 793 (1988).

[8] M.I. Dyakonov, A.L. Efros, D.L. Mitchell, Phys. Rev. 180, 813 (1969).

[9] E.N. Economou, M.H. Cohen, K.F. Freed, E.S. Kirkpatric, in: Amorphous and Liquid Semiconductors, Ed. J. Tauc, Plenum Press, London, New York 1974, p. 101.

[10] S.H. Koenig, R.D. Brown, III, W. Schillinger, Phys. Rev. 128, 1668 (1962).

[11] K.M. Mayer, J. Parisi, R.P. Huebener, Z. Phys. B, Condens. Malter 71, 171 (1988).

[12] T.O. Poehler, Phys. Rev. B 4, 1223 (1971).

[13] W. Rosner, G. Wuner, H. Herold, H. Ruder, J. Phys. B, At. Mol. Opt. Phys. 17, 29 (1984).

[14] J.F. Le Hir, J. de Phys. 28, 907 (1967).

[15] A.J. Shik, Fiz. Tech. Poluprovodn. 9, 872 (1975).

[16] G.M. Martin, A. Mitonneau, A. Mircea, Electron. Lelt. 13, 191 (1977).

[17] E. Kamińska, A. Piotrowska, W. Knap, P. Trautman, Acla Phys. Pol. A73, 501 (1988).

[18] K. Karpierz, M.L. Sadowski, M. Grynberg, in: Proc 20th Intern. Conf, on the Physics of Semiconductors, Saloniki 1990, Eds. E.M. Anastassakis, J.D. Joannopoullos, World Scientific, Singapore 1990, p. 609.

[19] B.A. Aronzon, I.M. Tsidilkovski, Phys. Stalus Solidi B 157, 17 (1990).

[20] J. Mycielski, Solid State Commun. 60, 165 (1986).

[21] E. Abrahams, P.W. Anderson, D.C. Licciardello, T.V. Ramakrishnan, Phys. Rev. Lett. 42, 637 (1979). 